\title{
Detection of intracranial aneurysms with unenhanced and echo contrast enhanced transcranial power Doppler
}

\author{
Carole L Turner, Peter J Kirkpatrick
}

\begin{abstract}
Objective-To assess transcranial power Doppler and the effect of an intravenous ultrasonic contrast agent for detecting intracranial aneurysms in patients admitted with a subarachnoid haemorrhage Methods-203 patients with subarachnoid haemorrhage were examined with the operator blind to the results of the admission CT and subsequent digital subtraction angiogram (DSA). In 98 cases patients were imaged using unenhanced transcranial power Doppler, and in 105 cases, patients were imaged both using unenhanced and echo contrast enhanced (Levovist, Schering AG) transcranial power Doppler.
\end{abstract}

Results-DSA detected 168 intracranial aneurysms in 128 patients (24 patients had multiple lesions) and 75 patients had an aneurysm negative DSA. There was agreement between DSA and transcranial power Doppler in 87 intracranial aneurysms of which 20 were only detected after infusion of contrast agent. Without contrast infusion, transcranial power Doppler showed a sensitivity of $40 \%$ and specificity of $91 \%$. A significant increase in detection was noted when using a contrast agent $\left(\chi^{2}=9.49, \mathbf{p}<0.001\right)$. With a contrast study the sensitivity increased to $55 \%$ with a specificity of $83 \%$. Intracranial aneurysm detection by transcranial power Doppler was significantly dependent on position using a contrast infusion $\left(\chi^{2}=15.87\right.$, $\mathbf{p}<0.05)$. A positive correlation was also found between the size of intracranial aneurysms measured by transcranial power Doppler and DSA $(r=0.55, \mathrm{p}<0.05)$. The transcranial power Doppler sensitivity and specificity increased with larger aneurysms and reached $100 \%$ for intracranial aneurysms greater than $12 \mathrm{~mm}$ diameter. Detection by transcranial power Doppler was also dependent on morphology $\left(\chi^{2}=14.46, p<0.001\right)$.

Conclusions-The detection of intracranial aneurysm by transcranial power Doppler is dependent on aneurysm location, size, and morphology and is enhanced with the use of an intravenous contrast agent.

(F Neurol Neurosurg Psychiatry 2000;68:489-495)

Keywords: transcranial power Doppler; intracranial aneurysm; subarachnoid haemorrhage; contrast agent
Subarachnoid haemorrhage from a ruptured intracranial aneurysm is associated with high morbidity and mortality despite improvements in surgery and medical support. ${ }^{12}$ By contrast, treatment for unruptured intracranial aneurysms is associated with a lower complication rate, the prevalence of which is about $1 \%{ }^{3}$ Although the natural history for unruptured intracranial aneurysms is unclear, the International Study into Unruptured Intracranial Aneurysms (ISUIA) has recently published data suggesting that the risk of rupture for aneurysms less than $10 \mathrm{~mm}$ in diameter is very low (about $0.05 \%$ a year) in patients with no history of subarachnoid haemorrhage. By contrast, the risk of rupture for patients with aneurysms $10 \mathrm{~mm}$ or more in diameter was much higher $(1 \%) .{ }^{4}$ Aneurysm size is therefore important when designing possible screening programmes.

To date, the identification of unruptured intracranial aneurysms depends on invasive digital subtraction angiography (DSA), magnetic resonance angiography (MRA), and CT angiography (CTA), all of which are expensive, and require specialist facilities and a high level of expertise. Each of these modalities shows varying degrees of detection, and none have $100 \%$ sensitivity. ${ }^{5-10}$ Digital subtraction angiography is still accepted as the gold standard for intracranial aneurysm detection with high resolution and associated anatomical detail, but attracts an associated morbidity (about $1 \%$ ) and mortality (about $0.5 \%) .{ }^{11-13}$ Therefore an attractive, rapid, and non-invasive means for identifying, localising, and characterising intracranial aneurysms, particularly those over $10 \mathrm{~mm}$ in size, may prove helpful for screening purposes and also for known lesions, both treated and untreated.

Non-invasive transcranial Doppler imaging with low frequency, high power output transcranial probes allows visualisation of the intracranial vessels ${ }^{14}$ and has been considered for imaging of intracranial aneurysms. Transcranial Doppler imaging is without significant side effects but visualisation of the intracranial vessels has been limited on account of temporal bone artefact and low anatomical resolution. About $16 \%$ to $20 \%$ of patients have a poor acoustic window preventing adequate insonation. ${ }^{15}$ These limitations have improved with the recent development of a "power Doppler" sequence that provides greater sensitivity to blood flow allowing good visualisation in most patients. 
Power Doppler is a relatively new technique in ultrasound imaging which detects the density of the red blood cells as opposed to their velocity. The Doppler frequency shift information is not utilised in power Doppler and is therefore not affected by aliasing. ${ }^{16}$ The technique is also virtually independent of angle and is therefore ideal for imaging of the intracranial vessels. The ability of transcranial power Doppler to image the intracranial vessels, and thus aneurysms, is highly dependent on the quality of the temporal bone window and partly accounts for the dependence of aneurysm detection in the specific locations. ${ }^{17}$

The resolution of blood flow and tissue properties can be further improved with ultrasonic contrast agents. Ries et al first showed the use of enhancing the transcranial Doppler signal intensity using galactose based microparticles. ${ }^{18}$ The contrast agent Levovist (Schering AG), consists of such microparticles (about $5 \mu \mathrm{m}$ diameter) which are coupled with palmitic acid $(0.1 \%)$, promoting stability for passage through the pulmonary vascular bed. The use of Levovist to enhance transtemporal imaging and resolution of vessels of the circle of Willis has obvious potential, but awaits clarification for aneurysm detection.

Previous studies have documented the ability of transcranial power Doppler to identify intracranial aneurysms with some prior knowledge of either the presence of an intracranial aneurysm, or the distribution and extent of blood seen on CT. ${ }^{19-23}$ The purpose of this study is primarily to evaluate the use of transcranial power Doppler in the overall detection and characterisation of intracranial aneurysms in patients who have had a subarachnoid haemorrhage and to assess the influence of an intravenous contrast agent (Levovist) in aneurysm detection.

\section{Subjects and methods}

Ethical approval was sought and approved by the Cambridge Local Research Ethics Committee.

\section{SELECTION OF PATIENTS}

A series of 203 patients ( 79 men, 124 women) were recruited into the study (mean age 55, range 20-85 years) over 18 months. All patients had subarachnoid blood confirmed by $\mathrm{CT}$ and a subsequent digital subtraction angiogram (DSA). Each patient was scanned by transcranial power Doppler with the operator blinded to the results of both CT and DSA.

\section{ULTRASOUND TECHNIQUE}

Using a Toshiba Powervision SSA-380 ultrasound machine with a multifrequency transcranial probe $(2-3 \mathrm{MHz})$, the circle of Willis was insonated through the temporal bone window (bilaterally) in both the coronal and transverse planes $^{21} 22$ and the occipital window for visualisation of the vertebral and basilar arteries. ${ }^{22}{ }^{24}$ Both conventional colour and power Doppler were employed; however, all aneurysms were measured in the power Doppler mode. For the purpose of this study, the calcified or thrombosed parts of an aneurysm were not included.
This was to compare directly with the measurements obtained by DSA. The major intracranial arteries comprising the circle of Willis were imaged and examined for possible aneurysms.

The contrast agent (Levovist $2.5 \mathrm{~g}$ ) was only available in the later part of the study. The initial 98 patients were therefore only examined with unenhanced transcranial power Doppler and the remaining 105 were imaged both before and after infusion of an ultrasonic contrast agent. An infusion protocol was employed for introduction of the contrast, as the bolus method was found to produce too short a period of enhanced signal (about 3 minutes). Using an infusion method, the controlled signal enhancement was extended by about 5 minutes, lasting 8-10 minutes, which allowed sufficient time to scan bilaterally. The contrast agent was administered intravenously through a 20 gauge Biovalve cannular using an IVAC P4000 anaesthesia syringe pump (Schering AG), with a $20 \mathrm{ml}$ syringe (provided with the contrast pack) and a $50 \mathrm{~cm}$ low pressure connector tube (Medrad, Inc). The optimal concentration was found to be $300 \mathrm{mg} / \mathrm{ml}$ at an infusion rate of $1 \mathrm{ml} / \mathrm{min}$. In two patients, the initial signal intensity was found to be sufficiently poor that a higher concentration of 400 $\mathrm{mg} / \mathrm{ml}$ was administered. This provided an infusion of $6 \mathrm{ml}$ and a corresponding time of enhanced signal lasting 5-6 minutes.

An aneurysm was defined by transcranial power Doppler where there appeared a blind ending "bulge" projecting from the parent vessel, where no normal artery would be expected. In the larger aneurysms the presence of bidirectional red and blue imaging in the colour duplex mode also aided identification. ${ }^{25}$ The presence of possible turbulent flow within the aneurysm was noted. When an aneurysm was suspected by transcranial power Doppler the image was frozen. With the aid of the cine loop, the size of an identified intracranial aneurysm was determined by the use of electronic calipers to draw around the outer edge of the colour signal of the aneurysm at the point of maximum size. Any suspected intracranial aneurysms were documented with respect to site and size before and after infusion of a contrast agent. Where possible, patients were imaged throughout contrast infusion (about 8 minutes), after which the aneurysm was either confirmed, or the presence of a previously unsuspected aneurysm was noted and documented. Blooming was reduced to a minimum by reducing the colour gain before commencing the infusion, and slowly increasing it throughout the study to maintain an optimal image.

Aneurysms detected by DSA were measured in three planes. The volume of the aneurysm was then determined, assuming an ovoid shape using the equation:

$$
\mathrm{V}=\frac{4}{3} \pi \times \frac{\mathrm{d}_{1}}{2} \times \frac{\mathrm{d}_{2}}{2} \times \frac{\mathrm{d}_{3}}{2}
$$

where $\mathrm{V}$ is the volume of the aneurysm and $d_{1}, d_{2}$, and $d_{3}$ are the diameters measured in 
three planes. The mean diameter $\left(\mathrm{d}_{\text {mean }}\right)$ was then calculated using the reverse equation:

$$
\mathrm{d}_{\text {mean }}=2 \times \sqrt[3]{\frac{3 \mathrm{~V}}{4 \pi}}
$$

For ultrasound measurements, the electronic caliper was used to delineate the circumference of the aneurysm in the best available plane, allowing the cross sectional area to be calcu-

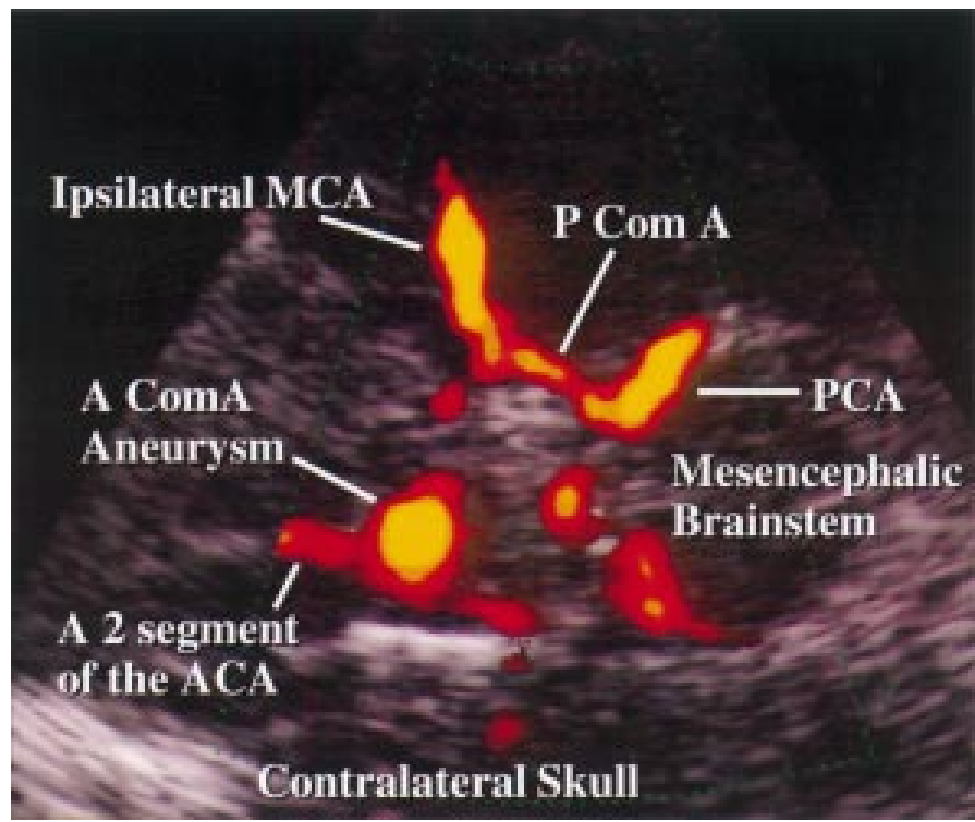

Figure 1 Transcranial power Doppler image of an anterior communicating artery aneurysm. A Com $A=$ anterior communicating artery; $P$ Com $A=$ posterior communicating artery; $P C A=$ posterior cerebral artery; $A C A=$ anterior cerebral artery; $M C A=$ middle cerebral artery.

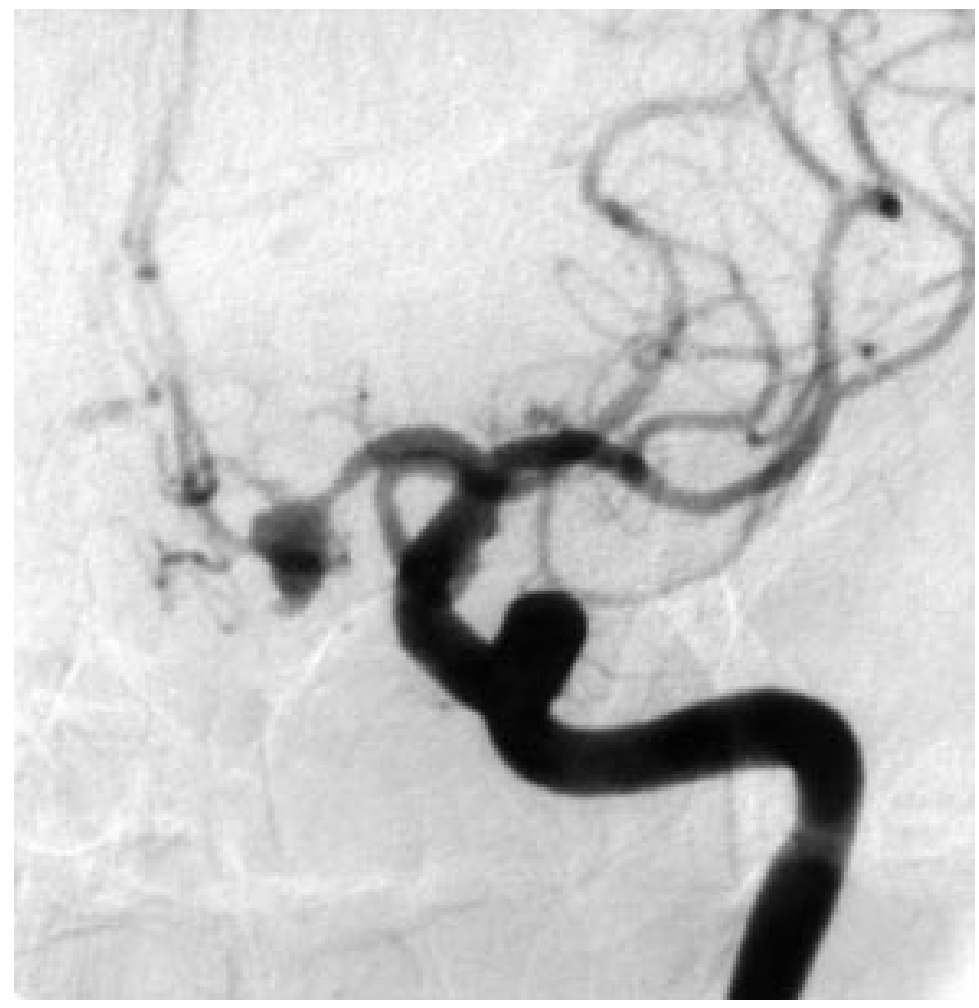

Figure 2 Corresponding angiographic image of the anterior communicating artery aneurysm.
Table 1 Effect of location on transcranial power Doppler detection of aneurysms

\begin{tabular}{lrc}
\hline & $D S A$ & Power Doppler \\
\hline A comm & 57 & $31(54)$ \\
P comm & 34 & $10(29)$ \\
MCA & 42 & $14(33)$ \\
Basilar & 10 & $6(60)$ \\
ICA & 11 & $5(45)$ \\
Peri & 9 & 0 \\
PICA & 3 & $1(33)$ \\
Ophth & 2 & 0 \\
Total & 168 & $67(40)$ \\
\hline
\end{tabular}

Numbers in parentheses represent percentage of aneurysms seen by transcranial power Doppler. A comm=anterior communicating artery; $\mathrm{P}$ comm=posterior communicating artery; $\mathrm{MCA}=$ middle cerebral artery; Peri=pericallosal artery; ICA=terminal internal carotid artery; PICA=posterior inferior cerebellar artery; Ophth=ophthalmic artery.

lated. The mean diameter was calculated using the equation:

$$
d=2 \times \sqrt{\frac{A}{\pi}}
$$

where $\mathrm{d}$ is diameter of the aneurysm and $\mathrm{A}$ is the cross sectional area. ${ }^{26}$

\section{STATISTICAL METHODS}

Analysis by $\chi^{2}$ test was used to compare the relation between aneurysms as determined by transcranial power Doppler and angiography and to evaluate the benefit of a contrast agent. Multivariable linear regression was used to compare sizes of aneurysms measured by transcranial power Doppler and angiography.

\section{Results}

COMPLICATIONS

Of the 203 patients who were entered into the study, 105 were imaged both with and without an infusion of ultrasonic contrast agent. Two patients refused the infusion study. No patients described significant side effects or an increase in their pre-existing symptoms as a result of the contrast agent infusion.

An image demonstrating an aneurysm positive patient is seen in figure 1 with the corresponding angiographic image figure 2 .

ANEURYSM DETECTION WITHOUT ECHO CONTRAST ENHANCEMENT

\section{Overall detection of intracranial aneurysms}

Out of 203 patients, digital subtraction angiography confirmed intracranial aneurysms in 128 patients (the remaining 75 had a negative DSA). In all patients who received treatment, the aneurysms were confirmed at surgery or during endovascular intervention. Digital subtraction angiography identified 168 intracranial aneurysms, with 24 patients having multiple lesions. In 13 patients the bone window was either poor or absent, resulting in an unsuccessful examination.

There was agreement between DSA and transcranial power Doppler in 67 intracranial aneurysms in terms of presence and location. Ultrasound showed a sensitivity of $40 \%$ and specificity $91 \%$ (seven false positive aneurysms were reported). For detection of aneurysm positive patients (single or multiple aneurysms), the corresponding figures were 53\% and $91 \%$. 
Table 2 Effect of size on transcranial power Doppler detection of aneurysms

\begin{tabular}{lrl}
\hline Size $(\mathrm{mm})$ & DSA & Power Doppler \\
\hline $2-3.9$ & 38 & $11(29)$ \\
$4-5.9$ & 49 & $13(27)$ \\
$6-7.9$ & 44 & $20(45)$ \\
$8-9.9$ & 14 & $7(50)$ \\
$10-11.9$ & 10 & $7(70)$ \\
$12-13.9$ & 1 & $1(100)$ \\
$14-15.9$ & 6 & $4(67)$ \\
$16-17.9$ & 2 & $2(100)$ \\
$18-19.9$ & 0 & 0 \\
$20-21.9$ & 1 & 0 \\
$22-23.9$ & 1 & $1(100)$ \\
$24-25.9$ & 2 & $67(40)$ \\
Total & 168 & \\
\hline
\end{tabular}

Numbers in parentheses represent percentage of aneurysms seen by transcranial power Doppler.

\section{Site of intracranial aneurysms}

The overall distribution of aneurysms detected by site is shown in table 1 . Aneurysm detection using transcranial power Doppler was heavily dependent on position, $\left(\chi^{2}=16.69, \mathrm{p}<0.05\right)$. The percentage of aneurysms found by transcranial power Doppler ranged from $60 \%$ of the basilar tip to $0 \%$ of pericallosal and ophthalmic aneurysms.

Effect of intracranial aneurysm size on detection The mean aneurysm size detected by transcranial power Doppler was $8.03 \mathrm{~mm}$ (range $2.5-24.7 \mathrm{~mm}$ ). The total distribution of aneurysm sizes detected by transcranial power Doppler is shown in table 2. Without a contrast study, ultrasound detected 16 out of 23 aneurysms greater than $10 \mathrm{~mm}(70 \%)$.

Comparison between DSA and transcranial power Doppler: intracranial aneurysm size

A significant correlation between the size of aneurysms measured by angiography and transcranial power Doppler was found $(\mathrm{y}=2.36 \mathrm{x}-$ 3.8, with $r=0.64, \mathrm{p}<0.01$ ) (fig 3). Ultrasound consistently underestimated the aneurysm size.

Effect of aneurysm morphology on intracranial aneurysm detection

Aneurysm detection by transcranial power Doppler was dependent on morphology $\left(\chi^{2}=9.72, p<0.01\right), 56 \%$ of the multiloculated

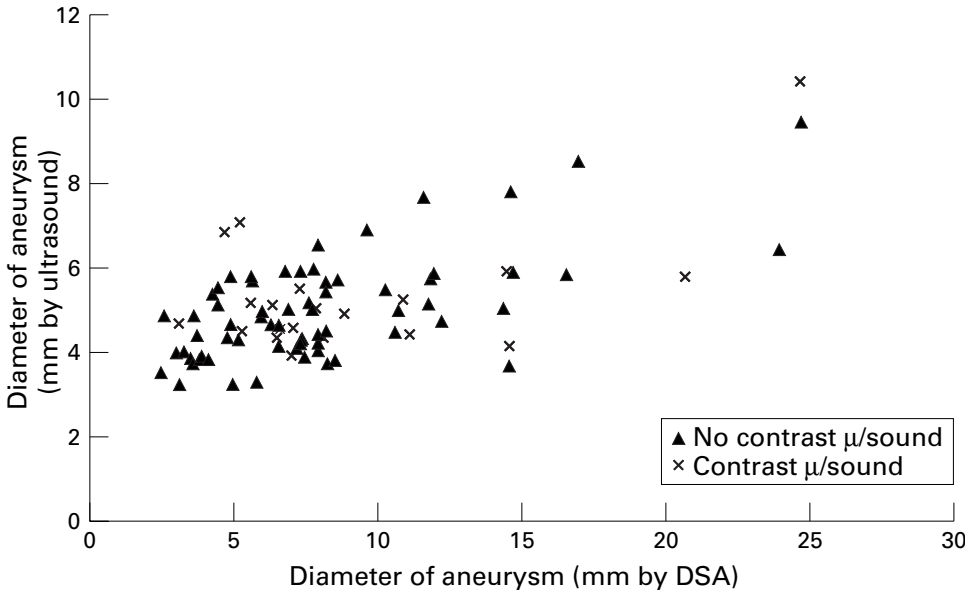

Figure 3 Aneurysms detected by transcranial power Doppler. Comparison of aneurysm sizes as determined by DSA and unenhanced transcranial power Doppler and by DSA and echo contrast enhancement.
Table 3 Effect of morphology on transcranial power Doppler detection of aneurysms

\begin{tabular}{lcc}
\hline & $D S A$ & Power Doppler \\
\hline Spherical & 92 & $33(36)$ \\
Multiloculated & 52 & $29(56)$ \\
Elongated & 24 & $5(21)$ \\
Total & 168 & $67(40)$ \\
\hline
\end{tabular}

Numbers in parentheses represent percentage of aneurysms seen by transcranial power Doppler.

aneurysms were identified by ultrasound whereas only $21 \%$ of the elongated aneurysms were detected (table 3 ).

\section{ANEURYSM DETECTION WITH ECHO CONTRAST} ENHANCEMENT

Overall detection of intracranial aneurysms

One hundred and five patients were imaged with and without a contrast agent; of these, 86 aneurysms were identified in 64 patients with DSA (the remaining 41 had a negative DSA). Four patients had a poor or absent bone window resulting in an unsuccessful examination. The detection of aneurysms before contrast infusion was $31 \%$ and after contrast $55 \%$ transcranial power Doppler identified 20 transcranial aneurysms only on infusion of a contrast agent.

With a contrast agent, ultrasound showed an increased sensitivity from $31 \%$ to $55 \%$, and reduced specificity from $93 \%$ to $83 \%$. For detection of aneurysm positive patients (single or multiple aneurysms), the corresponding figures increased from $39 \%$ to $67 \%$ for sensitivity, whereas specificity reduced from $93 \%$ to $83 \%$.

\section{Site of intracranial aneurysms}

The distribution of aneurysms detected by site is shown in table 4 . The detection was again heavily dependent on location $\left(\chi^{2}=15.87\right.$, $\mathrm{p}<0.05)$.

Effect of intracranial aneurysm size on detection The mean aneurysm size detected by transcranial power Doppler was $9.33 \mathrm{~mm}$ (range 3.11$24.73 \mathrm{~mm}$.). The total distribution of aneurysm sizes detected by transcranial power Doppler is shown in table 5 . Ultrasound detected $100 \%$ of aneurysms greater than $10 \mathrm{~mm}$ in the contrast study. Two of the largest aneurysms were only identified with a contrast infusion. For all aneurysms, there was a significant increase in detection using a contrast agent $\left(\chi^{2}=9.49\right.$, $\mathrm{p}<0.001)$. The total number of aneurysms detected both with and without an infusion of contrast is shown in fig 4 and is seen to be dependent on size.

Comparison between DSA and transcranial power Doppler: intracranial aneurysm size A positive correlation was seen between the size of aneurysms measured by angiography and transcranial power Doppler $(y=2.08 x-1.78$, with $r=0.55, \mathrm{p}<0.05)$. Again contrasted transcranial power Doppler consistently underestimated aneurysm size determined angiographically (fig 3). 
Table 4 Detection of aneurysms with echo contrast enhancement: effect of location

\begin{tabular}{lcccc}
\hline & \multicolumn{5}{c}{$\begin{array}{l}\text { Power Doppler } \\
\text { echocontrast } \\
\text { enhanced } \\
\text { (additional No } \\
\text { detected) }\end{array}$} & $\begin{array}{l}\text { Power Doppler } \\
\text { (total No detected) }\end{array}$ \\
\hline A comm & DSA & $\begin{array}{l}\text { Power Doppler } \\
\text { (unenhanced) }\end{array}$ & 9 & $22(79)$ \\
P comm & 28 & $13(46)$ & 3 & $5(33)$ \\
MCA & 15 & $2(13)$ & 4 & $11(50)$ \\
Basilar & 22 & $7(32)$ & 2 & $4(67)$ \\
ICA & 6 & $2(33)$ & 2 & $5(50)$ \\
Peri & 10 & $3(30)$ & 0 & 0 \\
PICA & 4 & 0 & 0 & $47(55)$ \\
Total & 1 & 0 & 20 & 0 \\
\hline
\end{tabular}

Numbers in parentheses represent percentage of aneurysms seen by transcranial power Doppler. For abbreviations see table 1 .

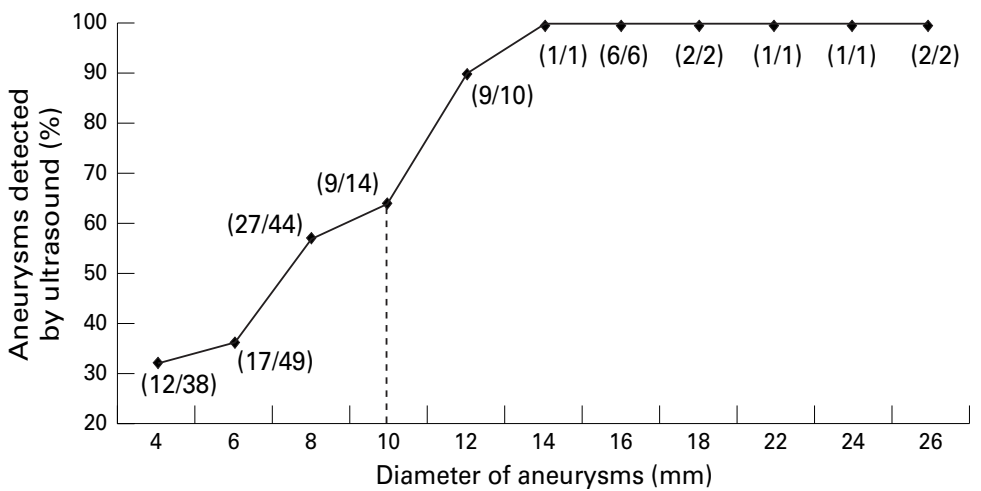

Figure 4 Effect of size on transcranial power Doppler aneurysm detection (all detected aneurysms). Numbers in parentheses represent transcranial power Doppler detected aneurysms/DSA detected aneurysms in each size epoch.

\section{Effect of aneurysm morphology on intracranial} aneurysm detection

The effect of morphology on detection rate is shown in table 6 , where the highest detection rate was still in the multiloculated aneurysms. Using a contrast agent, the effect of differing morphology on the detection by transcranial

Table 5 Detection of aneurysms with echo contrast enhancement: effect of size

\begin{tabular}{lcccc}
\hline Size (mm) & DSA & $\begin{array}{l}\text { Power Doppler } \\
\text { (unenhanced) }\end{array}$ & $\begin{array}{l}\text { Power Doppler } \\
\text { echo contrast } \\
\text { enhanced } \\
\text { (additional No } \\
\text { detected) }\end{array}$ & $\begin{array}{l}\text { Power Doppler } \\
\text { (total No detected) }\end{array}$ \\
\hline $2-3.9$ & 16 & $5(31)$ & 1 & $6(38)$ \\
$4-5.9$ & 26 & $7(27)$ & 4 & $11(42)$ \\
$6-7.9$ & 24 & $5(21)$ & 7 & $12(50)$ \\
$8-9.9$ & 8 & $4(50)$ & 2 & $6(75)$ \\
$10-11.9$ & 7 & $5(71)$ & 2 & $7(100)$ \\
$12-13.9$ & 3 & $1(33)$ & 2 & $3(100)$ \\
$14-15.9$ & 0 & 0 & 0 & $1(100)$ \\
$16-17.9$ & 1 & 0 & 1 & 0 \\
$18-19.9$ & 0 & 0 & 0 & $1(100)$ \\
$20-21.9$ & 0 & 0 & 1 & $47(55)$ \\
$24-25.9$ & 1 & 0 & 20 & 0 \\
Total & 86 & $27(31)$ & 0 & \\
\hline
\end{tabular}

Numbers in parentheses represent percentage of aneurysms seen by transcranial power Doppler.

Table 6 Detection of aneurysms with echo contrast enhancement: effect of morphology

\begin{tabular}{|c|c|c|c|c|}
\hline & $D S A$ & $\begin{array}{l}\text { Power Doppler } \\
\text { (unenhanced) }\end{array}$ & $\begin{array}{l}\text { Power Doppler } \\
\text { echo contrast } \\
\text { enhanced } \\
\text { (additional No } \\
\text { detected) }\end{array}$ & $\begin{array}{l}\text { Power Doppler } \\
\text { (total No detected) }\end{array}$ \\
\hline Spherical & 46 & $12(26)$ & 11 & $23(50)$ \\
\hline Multiloculated & 28 & $14(50)$ & 8 & $22(79)$ \\
\hline Elongated & 12 & $1(8)$ & 1 & $2(17)$ \\
\hline Total & 86 & $27(31)$ & 20 & $47(55)$ \\
\hline
\end{tabular}

Numbers in parentheses represent percentage of aneurysms seen by transcranial power Doppler. power Doppler increased in significance $\left(\chi^{2}=14.46, \mathrm{p}<0.001\right)$

\section{Discussion}

This study has shown that the detection of intracranial aneurysms by transcranial power Doppler is highly dependent on the size of the aneurysm, with anatomical position and morphology also determining factors. Ultrasonic contrast agent significantly increased the detection rate.

The detection rates in this study are not comparable with some previous studies. However, most published papers on aneurysm detection rates have been performed with either knowledge of the results of other imaging investigations (CT or MRI) or had chosen a selected population of subarachnoid haemorrhage (only aneurysm positive patients with subarachnoid haemorrhage were included). Becker et $a l^{14}$ detected $76 \%$ of aneurysms, which was higher than that detected by angiography $(73 \%)$. The detection rate of Klotzsch et $a l^{11}$ was, however, comparable with our study $(47 \%)$ despite the prior knowledge of the presence of an aneurysm.

Despite the superficial location of middle cerebral artery aneurysms, a relatively low number were detected. This is, in part, due to the difficulty in isolating the aneurysm from adjacent vessels, as vessels at the bifurcation can either imitate or mask an aneurysm. However, a high percentage of basilar tip aneurysms were detected, where the posterior cerebral arteries can usually be seen separate from the basilar tip. None of the pericallosal aneurysms were detected, as the pericallosal arteries are outside the field of view through the temporal bone window. Ophthalmic aneurysms are often hidden from the acoustic window due to the surrounding dense bone. The internal carotid artery and anterior and posterior communicating artery aneurysms attracted an intermediate level of detection (33\%-79\%).

Previous studies have shown that a contrast agent improves the visualisation of the intracranial arteries. ${ }^{27}$ The increased sensitivity provided by a contrast agent may be of particular importance for large aneurysms with low flow velocities (as shown by the two aneurysms above $10 \mathrm{~mm}$ in diameter which were only imaged after an infusion of contrast) and for patients with poor acoustic windows.

It is difficult to explain why the specificity decreased in the contrast enhanced study. However, all patients in the second half of the study were examined with echo contrast enhancement (apart from two who refused consent), irrespective of the quality of the native image. Where the acoustic bone window had provided a good image in the unenhanced examination, the use of a contrast agent caused an excess in the signal which actually degraded the image and some false positive aneurysms were diagnosed.

The use of the infusion method for administering a contrast agent allows for a prolonged enhancement of the image to about 8 minutes. This represents an improvement over the bolus method and is a more controlled method of 
administration. Despite contrast infusion, not all aneurysms were detected. The ability of a contrast agent to enhance the image of an aneurysm may depend on the flow patterns within the aneurysm. Thus the concentration of microbubbles within a given aneurysm depends on several independent factors, including size, shape, and position with respect to the parent arteries although the size of the aneurysm does not necessarily dictate the flow velocity within it. Flow characteristics may therefore be important in aneurysm detection and be partly responsible for false negatives.

The overall detection rate of aneurysms above $10 \mathrm{~mm}$ diameter by ultrasound was $96 \%$. This has possible clinical relevance in terms of the treatment of unruptured aneurysms. In patients with no history of subarachnoid haemorrhage, the cumulative rate of rupture of aneurysms less than $10 \mathrm{~mm}$ is about $0.05 \%$ per year. By contrast, the rupture rate of aneurysms greater than $10 \mathrm{~mm}$ is 20 times higher, ${ }^{4}$ a finding which has important implications for a screening programme targeting high risk patients. ${ }^{38}{ }^{29} \mathrm{Six}$ aneurysms greater than $10 \mathrm{~mm}$ diameter were detected only after an infusion of a contrast agent. All of these patients had a poor acoustic window and before the infusion none or few intracranial vessels were visualised.

A significant relation was found between the size of the aneurysms as measured by DSA and transcranial power Doppler, in agreement with a previous study ${ }^{21}$; however, transcranial power Doppler consistently underestimated the size. The difficulty in comparing aneurysm sizes by DSA and ultrasound is compounded by the different imaging planes. Aneurysms are imaged by DSA in three planes and the maximum diameter in each plane can be measured. By contrast, due to the restriction of the bone window reproducing comparable views using transcranial power Doppler is not possible. Although aneurysms are often irregular in outline, in this study they were measured in both modalities assuming a circular or ovoid shape and thus allowing a similar degree of inaccuracy of measurement. Aneurysms elongated in the anteroposterior plane would hence always be underestimated in size by transcranial power Doppler.

Different flow patterns may also explain why transcranial power Doppler underestimated the size of aneurysms. The use of transcranial power Doppler in evaluating the expansibility of aneurysms with the cardiac cycle has been documented. ${ }^{30}$ Flow within an aneurysm may vary dramatically and aneurysms imaged during diastole can actually disappear from the image. ${ }^{19}$ This is almost certainly a reflection of blood flow pattern rather than anatomical collapse of the aneurysm. Therefore, if the flow is significantly reduced around the aneurysm border, detection with transcranial power Doppler will underestimate aneurysm size. The aneurysms identified with contrast showed a poorer correlation than those measured without. Although the numbers involved were too low for a sensible statistical comparison, an explanation may be derived from the known artefacts of contrast agents-namely, aliasing and enhanced variation during the cardiac cycle.

The detection rate of ultrasound with respect to aneurysm morphology without contrast infusion was seen to be $36 \%$ and $56 \%$ respectively for spherical and multiloculated aneurysms. It was somewhat lower for the elongated aneurysms (21\%). This is possibly because these could be incorrectly mistaken for vessels, whereas the multiloculated and spherical aneurysms can be more readily resolved from the surrounding vessels. This was the case for an $11.9 \mathrm{~mm}$ elongated aneurysm, which was undetected by transcranial power Doppler. The ratio of the detection rate based on morphology remained the same in the contrast study. The highest detection of the multiloculated aneurysms was possibly due to the pathological morphology involving several imaging planes.

We are presently collecting data on unruptured aneurysms. Since the conception of this study, ISUIA has reported that only aneurysms greater than $10 \mathrm{~mm}$ in diameter are relevant. ${ }^{4}$ As the sensitivity in our study approaches $100 \%$ for these aneurysms, there is relevance in screening for large, unruptured aneurysms and for surveillance of such aneurysms. This study confirms the findings of previous work, ${ }^{21}$ in which the rate of aneurysm detection by transcranial power Doppler is not sufficient at present to be applied as an indiscriminate screening tool. With the use of contrast agents, transcranial power Doppler may be valuable in the detection or monitoring of large intracranial aneurysms, which attract treatment considerations. Further experience is required to assess the accuracy of transcranial power Doppler both with and without the use of novel contrast agents. The potential for imaging aneurysms and the intracranial vessels has been demonstrated. This may prove particularly useful for the selective monitoring of known aneurysms in specific locations, and also for the detection of aneurysm refilling after endovascular treatment.

This study was funded by an Anglia and Oxford research and development grant ref: RCC56267.

1 Schievink WI, Wijdicks EFM, Parisi JE, et al. Sudden death from aneurysmal subarachnoid haemorrhage. Neurology 1995;45:871-4

2 Ingall TJ, Whisnant JP, Wiebers DO, et al. Has there been a decline in subarachnoid haemorrhage mortality? Stroke 1989;20:718-24.

3 Schievink WI. Intracranial aneurysms. N Engl f Med 1997; 336: $28-40$.

4 International Study of Unruptured Intracranial Aneurysms Investigators. Unruptured intracranial aneurysms: risk of rupture and risks of surgical intervention. $N$ Engl $\mathcal{F ~ M e d ~}$ 1998;339:1725-33.

5 Gouliamos A, Gotsis E, Vlahos L, et al. Magnetic resonance angiography compared to intra-arterial digital subtraction angiography in patients with subarachnoid haemorrhage. Neuroradiology 1992;35:46-9.

6 Alberico RA, Patel M, Casey S, et al. Evaluation of the Circle of Willis with three-dimensional CT angiography in patients with suspected intracranial aneurysms. $\mathrm{Am} \mathcal{F ~ N e u -}$ roradiol 1995;16:1571-8.

7 Schwartz RB, Tice HM, Hooten SM, et al. Evaluation of cerebral aneurysms with helical CT: correlation with conventional angiography and MR angiography. Neuroradiology 1994;192:717-22.

8 Katz DA, Marks MP, Napel SA, et al. Circle of Willis: evaluation with spiral CT angiography, MR angiography, and conventional angiography. Radiology 1995;195:445-9. 
9 Strayle-Batra M, Skalej M, Wakhloo AK, et al. ThreeStrayle-Batra M, Skalej M, Wakhloo AK, et al. Threedimensional spiral CT angiography in the detection

10 Baumgartner RW, Mattle HP, Aaslid R. Transcranial colorcoded duplex sonography, magnetic resonance angiography and computerised tomography angiography: methods, applications, advantages and limitations. If Clin Ultrasound 1995;23:89-111.

11 Heiserman JE, Dean BL, Hodak JA, et al. Neurological complications of cerebral angiography. Am $\mathcal{f}$ Neuroradio 1994;15:1401-7.

12 Waugh JR, Sacharias N. Arteriographic complications in the DSA era. Radiology 1992;182:243-6.

13 Dion JE, Gates PC, Fox AJ, et al. Clinical events following neuroangiography: a prospective study. Stroke 1987;18: 997-1004.

14 Becker G, Greiner K, Kaune B, et al. Diagnosis and monitoring of subarachnoid haemorrhage by transcranial monitoring of subarachnoid haemorrhage by transcranial color-coded

15 Seidel G, Kaps M, Gerriets T. Potential and limitations of transcranial color-coded sonography in stroke patients. transcranial color-code

16 Forsberg F, Goldberg BB. Ultrasound contrast agents. In Goldberg BB, ed. New imaging techniques with ultrasound contrast agents. London: Marin Dunitz, 1977:177-92.

17 Postert T, Federlein J, Przuntek H, et al. Insufficient and absent acoustic temporal bone window: potential and limitations of transcranial contrast-enhanced color-coded sonography and contrast-enhanced power-based sonography. Ultrasound Med Biol 1997;23:857-62.

18 Ries F, Kaal K, Schultheiss R, et al. Air microbubbles as a contrast medium in transcranial Doppler sonography. $f$ Neuroimaging 1991;1:173-8.

19 Wardlaw JM, Cannon JC. Color transcranial power Dopple ultrasound of intracranial aneurysms. F Neurosurg 1996;84: 459-61.
20 Martin PJ, Gaunt ME, Naylor AR, et al. Intracranial aneurysms and arteriovenous malformations: transcranial
colour-coded sonography as a diagnostic aid. Ultrasound colour-coded sonography

21 KlotzschC, Nahser HC, Fischer B, et al. Visualisation of intracranial aneurysms by transcranial duplex sonography. Neuroradiology 1996;38:555-9.

22 Baumgartner RW, Mattle HP, Kothbauer K, et al. Transcranial color-coded duplex sonography in cerebral aneurysms. Stroke 1994;25:2429-34.

23 Griewing B, Motsch L, Piek J, et al. Transcranial power mode Doppler duplex sonography of intracranial aneurysms. F Neuroimaging 1998;8:155-8.

24 Kaps M, Seidel G, Bauer T, et al. Imaging of the intracranial vertebrobasilar system using color-coded ultrasound. Stroke 1992;23:1577-82.

25 Bogdahn U, Becker G, Schlachetzki F. Echoenhancers and transcranial color duplex sonography. Berlin: Blackwell, 1998.

26 Brown B, Hodge JL, Howat RD, et al. Maths in action, 2nd edition. China: Thomas Nelson, 1995.

27 Postert T, Federlein J, Przuntek H, et al. Comparison of transcranial power Doppler and contrast-enhanced colourcoded sonography in the identification of intracranial

28 Nakagawa T, Hashi K. The incidence and treatment of asymptomatic unruptured cerebral aneurysms. F Neurosurg 1994;80:217-23.

29 Wiebers DO, Torres VE. Screening for unruptured aneurysms in autosomal dominant kidney disease. $N$ Engl f Med 1992;327:953-5.

30 Wardlaw JM, Cannon JC, Statham PFX, et al. Does the size of intracranial aneurysms change with intracranial pressure? Observations based on color power transcranial Doppler ultrasound. $\mathcal{F}$ Neurosurg 1998;88:846-50.

31 Percival J, Wardlaw JM, Cannon JC. Observer variability in the measurement of the size of intracranial aneurysms using power TCD. 7 Neuroimaging 1998;8:75-7. 